\title{
THE ROUTE PROBLEM OF MULTIMODAL TRANSPORTATION WITH TIMETABLE UNDER UNCERTAINTY: MULTI-OBJECTIVE ROBUST OPTIMIZATION MODEL AND HEURISTIC APPROACH
}

\author{
Yong Peng*, Pengcheng Yong and Yijuan Luo
}

\begin{abstract}
The uncertainty of transportation duration between nodes is an inherent characteristic and should be concerned in the routing optimization of the multimodal transportation network to guarantee the reliability of delivery time. The interval number is used to deal with the uncertainty of transportation duration, and the multi-objective robust optimization model is established which covers the transportation duration and the cost. To solve the combinatorial optimization problem of this study, Non-Dominated Sorting Genetic Algorithm-II (NSGA-II) is designed, which integrates the $(\mu+\lambda)$ selection method elite retention and the external filing elite retention. Our findings verify the efficiency of the proposed approach by analyzing the diversity, distribution and convergence of the frontier solutions. Finally, near-optimal solutions are obtained with the proposed algorithm in the numerical example. The present study can provide decision reference for multimodal transportation carriers in making transportation plan under uncertainty.
\end{abstract}

Mathematics Subject Classification. 90B06.

Received February 9, 2020. Accepted September 28, 2020.

\section{INTRODUCTION \& LITERATURE REVIEW}

The advanced stage of transportation development is multimodal transportation [18,27]. Routing optimization is one of the important research directions of multimodal transportation $[5,12,32]$, which is also the extension and expansion of the classic shortest path problem [3,25,29]. Combining the real transportation environment with an innovative approach to improve transportation efficiency, reduce transportation cost and satisfy customer requirements is the core of the path optimization problem.

In the previous literature, multimodal transportation route optimization has been extensively studied [26]. Most multimodal transportation route optimization problems were developed with a single objective under certain conditions $[28,34,37]$. However, the requirements of customers and transportation enterprises were often diverse and even conflicting. For example, some customers preferred the lowest freight rates while some others would rather pay more for faster delivery or a certain person expected to cut transportation cost and duration simultaneously $[23,38]$. This means that it cannot depend on a single objective and should be considered as a multi-objective optimization problem $[4,13,15,31]$. In general, there were two conventional methods for

Keywords. Multimodal transportation, multi-objective, route optimization, uncertainty, NSGA-II.

School of Transpotation, Chongqing Jiaotong University, Chongqing 400074, P.R. China.

*Corresponding author: pyepeng@163.com 
addressing multi-objective optimization problems, which are the $\epsilon$-constraint method and the weighted sum method $[6,20,39]$.

In the complex transportation environment, it is more significant to take the uncertainty factors of multimodal transportation into account. Resat and Turkay [23] proposed a mixed-integer optimization model that considered traffic congestion of the routes among nodes that account for the transportation duration uncertainty. Assadipour et al. [2] considered the uncertainty of transportation duration caused by the congestion of the intermodal yards in the railtruck intermodal transportation of hazardous materials. In this regard, the risk uncertainty related to the placement of transfer yards as well as the possibility of disruptions at those facilities was concerned with the intermodal transportation of hazardous materials by Ghaderi et al. [11]. Meanwhile, information uncertainties were discussed in the modeling and optimization of railtruck system intermodal transportation by Wang et al. [30]. In addition to considering the uncertainties of using cost, the capacities of terminals and transportation cost also were observed in the works of Abbassi et al. [1].

There are various researches for the uncertainty of multi-objective multimodal transportation route optimization. Demir et al. [8] studied the design of green intermodal transportation service network problems with transportation duration as well as demands uncertainties for combined offline intermodal routing decisions. According to their formulation, a stochastic approach of the sample average approximation method was adopted to generate robust transportation plans. Hrusovsky et al. [14] further extended the study by improving the algorithm. The paper innovated a novel solution framework to support the operational-level decisions by using a simulation model that includes stochastic elements. It should be noted that the approach of fuzzy logic was also frequently employed to deal with uncertainty problems. Yang et al. [35] considered the uncertainty of transportation duration and cost as well as developed a comprehensive hybrid methodology of combining the Fuzzy Random Simulation (FRS) technique and Multi-start Simulated Annealing (MSA) algorithm to achieve nearoptimal solutions in a reasonable time for the proposed problem. Fazayeli et al. [9] studied the location routing decision-making issue in the multimodal transportation network and established a mixed-integer mathematical fuzzy model. Time window constraints and fuzzy numbers were imposed upon the formulation to represent the uncertainty of transportation duration and demand respectively. Additionally, metaheuristic approaches have gained attention in dealing with NP-hard Problem in the context of multimodal transportation network route optimization with uncertainty elements. A new method of handling multi-objective based on Fire-Works Algorithm (FWA) was introduced by Mnif et al. [21], which aimed to determine the shortest and efficient itinerary of satisfying a certain set of demands and operational constraints. Wang et al. [30] adopted a Memetic Algorithm (MA) to solve the problem of the hub-and-spoke based road-rail intermodal transportation network design and obtained high-quality solutions. Abbassi et al. [1] developed an effective hybrid solution approach combining the Population-Based Simulated Annealing (PBSA) with an exact method to achieve near-optimal solutions in a reasonable time for a real network case study.

Rail and water transportation usually have a fixed departure timetable [16], When the goods arrived at the transfer nodes and completed the loading and unloading, it might not be transported immediately due to the timetable limitation of the next mode of transportation [33]. Liu et al. [17] indicated that timetable would affect the choice of transportation routes and modes. So it makes more sense to consider timetable in multimodal transportation.

Table 1 outlines a taxonomy of relevant research on multimodal transportation route optimization in recent years and the taxonomy contains five major categories in terms of models and algorithms that further explain the studies differences. As can be found from Table 1, multi-objective optimization is often considered in the previous studies, but there are few attention about the some more realistic aspects that could be expressed as follows: Frist, there few formulations integrated the time uncertainty of transportation and the influence of timetable for trains and barges in the route optimization of multimodal transportation simultaneously. Second, in most literature, the method of dealing with uncertainty is to use an accurate probability distribution or fuzzy membership function with more reliable prior data. However, the reliable prior data are often difficult to obtain and irregular [36]. Third, in the problem solving, there is less literature applied heuristic algorithms combined with non-dominance theory to deal with multi-objective optimization, Furthermore, NSGA-II is 
TABLE 1. Some related papers.

\begin{tabular}{|c|c|c|c|c|c|c|}
\hline \multirow{2}{*}{ Paper } & \multirow{2}{*}{ Uncertainty type } & \multirow{2}{*}{ Uncertain para. exp. } & \multirow{2}{*}{ TB } & \multirow{2}{*}{ Obj. } & \multicolumn{2}{|c|}{ Treatment method } \\
\hline & & & & & For obj. & For alg. \\
\hline$[32]$ & - & - & - & $\mathrm{C}, \mathrm{T}$ & SW & Exact \\
\hline [28] & - & - & - & $\mathrm{C}$ & - & B\&B \\
\hline$[34]$ & - & - & - & $\mathrm{C}$ & - & Goal program \\
\hline$[23]$ & $\mathrm{T}$ & - & $\checkmark$ & $\mathrm{C}, \mathrm{T}$ & A_ $\epsilon$ & Exact \\
\hline$[13]$ & - & - & - & $\mathrm{C}$ & - & $\mathrm{DP}$ \\
\hline$[15]$ & - & - & - & $\mathrm{C}, \mathrm{T}$ & ND & Cplex \\
\hline$[6]$ & - & - & - & $\mathrm{C}, \mathrm{T}$ & SW & $\mathrm{DP}$ \\
\hline$[39]$ & $\mathrm{C}, \mathrm{T}$ & $\mathrm{RN}$ & - & $\mathrm{C}, \mathrm{T}$ & C_c & Exact \\
\hline$[2]$ & $\mathrm{T}$ & - & - & $\mathrm{C}, \mathrm{R}$ & ND & NSGA-II \\
\hline$[30]$ & Demand,C,T & TFN & - & $\mathrm{C}, \mathrm{T}$ & SW & MA \\
\hline [1] & Capacity,C & IN & - & $\mathrm{C}$ & - & PBSA \& Exact \\
\hline$[8]$ & Demand, $\mathrm{T}$ & $\mathrm{RN}$ & - & $\mathrm{C}, \mathrm{T}, \mathrm{E}$ & SW & SAA \\
\hline [14] & $\mathrm{T}$ & $\mathrm{RN}$ & - & $\mathrm{C}, \mathrm{T}, \mathrm{E}$ & SW & HS \\
\hline$[35]$ & $\mathrm{C}, \mathrm{T}$ & $\mathrm{TFN}$ & - & $\mathrm{C}, \mathrm{T}$ & SW & MSA \\
\hline$[9]$ & Demand,T & $\mathrm{TFN}$ & - & $\mathrm{C}$ & - & Two-part GA \\
\hline [21] & - & - & - & $\mathrm{C}, \mathrm{T}$ & ND & FWA \\
\hline$[33]$ & - & - & $\checkmark$ & $\mathrm{C}, \mathrm{R}$ & SW & B\&B \\
\hline$[17]$ & $\mathrm{T}$ & IN & $\checkmark$ & $\mathrm{C}$ & - & GA \\
\hline This paper & $\mathrm{T}$ & IN & $\checkmark$ & $\mathrm{C}, \mathrm{T}$ & ND & NSGA-II \& EFER \\
\hline
\end{tabular}

Notes. T: time, C: cost, exp.: expression, RN: random number, TFN: Triangular fuzzy number, IN: Interval number, TB: Timetable, R: risk, E: environment, SW: Sum weighting, A_ $\epsilon$ : Augmented $\epsilon$-constraint, C_c: chance constraint, ND: Non-dominance theory, B\&B: Branch\&Bound, DP: Dynamic program, SAA: Sample average approximation, HS: Hybrid simulation-optimization, GA: Genetic algorithm, EFER: External filing elite retention.

a common multi-objective optimization evolutionary algorithm, while the merits and demerits of elite retention strategies in NSGA-II has not been discussed in any of the previous studies of multimodal transportation route optimization. This study is an effort to bridge the stated gaps with contributions in both model formulation and solution methodology.

- The impact of timetable on transportation mode is analyzed and making a detailed description in the mathematical model;

- Interval numbers are used to indicate uncertain transportation time, and introduced robust control parameters to analyze the uncertainty characteristics;

- Designing a non-dominated genetic algorithm with external archiving strategy to solve the proposed problem and the performance of the algorithm and the quality of the solution are evaluated.

The rest of this paper is organized as follows. Following the introduction \& literature review, a description of the problem and its formulation are presented in Section 2. The solution methodology is presented in Section 3. Algorithms analysis and numerical results for proposal problem instances are reported in Section 4. Finally, Section 5 concludes the paper. 


\section{PROBlem DESCRIPTION}

In this paper, we consider a multimodal transportation network including three modes of the highway, railway and waterway, and more than two available modes could be selected between origin node and destination. However, road transportation duration is often influenced by congestion and unexpected accidents. Extreme weather and port congestion are important reasons for the uncertainty of shipping duration. Besides, waterway and railway transportation timetable limitations also affect the goods departure time to the next node after reload. The routing decision of our model which aims to simultaneously minimize the total transportation cost and duration between the origin node and destination is based on some assumptions given as follows:

(1) Only a batch of goods and not allow to spill in transportation;

(2) The transfer duration and cost between different modes of transportation are known;

(3) The arrival time of goods is the start time of transferring;

(4) The time of goods leave for the next node is the latest departure time from the timetable of the selected transportation mode after finished loading and unloading;

(5) Sufficient capacity of transportation and transshipment facilities.

\subsection{Index set}

In this study, we assume a multimodal transportation network $G=(N, E, M)$ where $N$ is the set of nodes

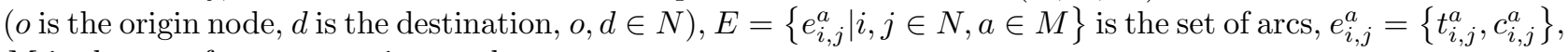
$M$ is the set of transportation modes.

\section{Parameters}

C The total cost of transportation;

$T$ The total duration of transportation;

$\delta_{i} \quad$ The time that the goods finished transshipment at the node $i$;

$c_{i, j}^{a} \quad$ The transportation cost of one unit of good from node $i$ to $j$ with $a$ transportation mode selected;

$t_{i, j}^{a} \quad$ The transportation duration from node $i$ to $j$ with $a$ transportation mode selected;

$T_{i}^{G} \quad$ The goods depart time from the node $i$, and the goods departure time of node $o$ is known;

$T_{i}^{R} \quad$ The time that the goods arrive at the node $i$;

$\tilde{t}_{i, j}^{a} \quad$ The Interval Number of transportation duration from node $i$ to $j$ with $a$ transportation mode selected;

$\Gamma_{i, j}^{a} \quad$ The timetable corresponding to transportation mode $a$ from node $i$ to $j$, it has been known in advance and can be defined as $\Gamma_{i, j}^{a}=\left(\ldots, \Phi_{n-1}, \Phi_{n}, \ldots\right)$, and $\Phi_{n}$ represents the departure time of $n$ shift.

$\theta_{i}^{a, b} \quad$ The cost on the changing of transportation mode from $a$ to $b$ at the node $i$;

$\tau_{i}^{a, b} \quad$ The transshipment duration of transportation mode from $a$ to $b$ at the node $i$;

$\hbar_{i, j}^{a} \quad$ The interval robust control parameter from node $i$ to $j$ with $a$ transportation mode selected.

\section{Decision variable}

$$
x_{i, j}^{a}= \begin{cases}1, & \text { if the transportation mode a is selected from node } i \text { to } j \\ 0, & \text { otherwise. }\end{cases}
$$

Set $U=\left\{(i, j, a) \mid x_{i, j}^{a}=1\right\}, W=\left\{(j, a, b) \mid x_{i, j}^{a}=1 \& x_{j, k}^{b}=1\right\}$. Equation (2.1) indicates how to obtain the departure time of node $i$ according to the time $\delta_{i}$ and the timetable $\Gamma_{i, j}^{a}$.

$$
\varphi\left(\delta_{i}, \Gamma_{i, j}^{a}\right)=\Phi_{n}, \Phi_{n-1}<\delta_{i} \leq \Phi_{n}
$$




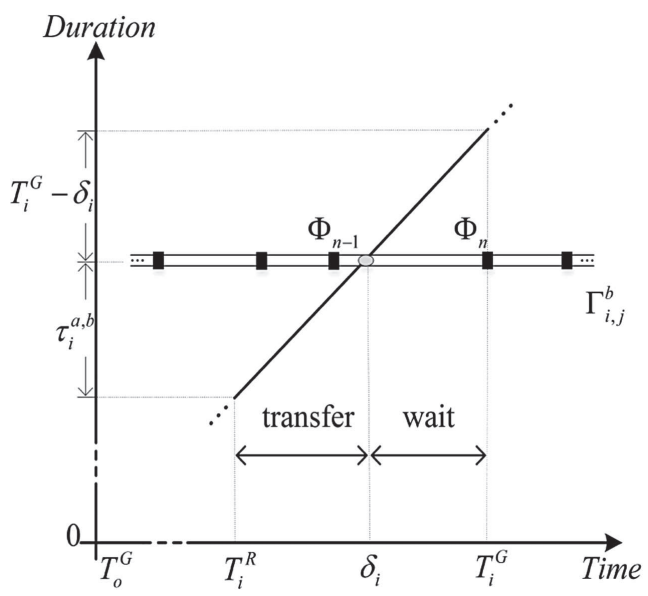

FIgURE 1. The transshipment duration in the node $i$.

\subsection{Interval uncertainty and robust control}

The Interval Number represents a kind of uncertainty, which is composed of a pair of ordered real numbers, with the dual characteristics of set and number value [22]. This $\widetilde{t}_{i, j}^{a}=\left[t_{i, j}^{a-}, t_{i, j}^{a+}\right]=\left\{t_{i, j}^{a} \mid t_{i, j}^{a-} \leq t_{i, j}^{a} \leq t_{i, j}^{a+}\right\}$ represents the uncertainty of the transportation duration from node $i$ to $j$ with $a$ transportation mode selected. "-" and "+" represents the lower bound of interval and the upper bound of interval respectively. Equation (2.2) indicates the uncertainty relationship between the control parameter $\hbar_{i, j}^{a}$ and the transportation time $t_{i, j}^{a}$ in the interval.

$$
\hbar_{i, j}^{a}=\frac{t_{i, j}^{a}-t_{i, j}^{a-}}{t_{i, j}^{a+}-t_{i, j}^{a-}}, \quad \forall i, j \in N, \forall a \in M
$$

\subsection{Multi-objective multimodal transportation robust optimization model}

As shown in Figure 1, the transshipment duration in the node $i$ under the timetable limitation includes the transferring duration and the waiting duration considering the timetable. The $\delta_{i}$ can be formulated as follow:

$$
\delta_{i}=T_{i}^{R}+\tau_{i}^{a, b}, \quad \forall(i, a, b) \in W .
$$

The $T_{i}^{G}$ can be formulated as follow:

$$
T_{i}^{G}=\varphi\left(\delta_{i}, \Gamma_{i, j}^{b}\right), \quad \forall(i, j, b) \in U .
$$

The $T_{j}^{R}$ can be formulated as follow:

$$
T_{j}^{R}=T_{i}^{G}+\hbar_{i, j}^{a}\left(t_{i, j}^{a+}-t_{i, j}^{a-}\right)+t_{i, j}^{a-}, \quad \forall(i, j, b) \in U .
$$

In this paper, we propose a bi-objective optimization model. The first objective seeks to minimize the total cost of multimodal transportation, including the itinerary and transshipment cost. The second objective seeks to minimize the total duration of multimodal transportation. A robust optimization method based on control parameters $\hbar_{i, j}^{a}$ is employed to solve the problem. (The constraints given above are not repeated in the following models.)

$$
\begin{aligned}
\min T & =T_{d}^{R}-T_{o}^{G} \\
\min C & =\sum_{i \in N} \sum_{j \in N \backslash\{i\}} \sum_{a \in M} c_{i, j}^{a} x_{i, j}^{a}+\sum_{(i, a, b) \in W} \theta_{i}^{a, b}
\end{aligned}
$$


s.t.

$$
\begin{aligned}
& \sum_{j \in N \backslash\{i\}} \sum_{a \in M} x_{i, j}^{a} \leq 1, \quad \forall i \in N \\
& \sum_{i \in N \backslash\{0\}} \sum_{a \in M} x_{o, i}^{a}=\sum_{i \in N \backslash\{d\}} \sum_{a \in M} x_{i, d}^{a}=1 \\
& \sum_{i \in N \backslash\{o\}} \sum_{a \in M} x_{i, o}^{a}=\sum_{i \in N \backslash\{d\}} \sum_{a \in M} x_{d, i}^{a}=0 \\
& \sum_{i \in N \backslash\{j\}} \sum_{a \in M} x_{i, j}^{a}=\sum_{i \in N \backslash\{j\}} \sum_{a \in M} x_{j, i}^{a}, \quad \forall j \in N \backslash\{o, d\} .
\end{aligned}
$$

Equation (2.8) means the selection of mode, that one mode (transportation route) can be selected between two nodes. If it is zero, it means that this node $i$ is not included in the transportation. Equations (2.9) and (2.10) ensure that the goods depart from the origin node $o$ to the destination $d$; Equation (2.11) represents that the conservation of flow at the node $j$ that requires the flow originating at the node $j$ to equal the flow entering the node $j$.

\section{The NSGA-II SOLUtion METhodology}

The solution space of our proposed problem is discontinuous, and GA can directly deal with the discrete property of the problem [19]. The NSGA-II, as the variant of genetic algorithm, is one of the most popular multi-objective evolution algorithms with fast running speed and good convergence advantages [7]. For these reasons, NSGA-II is used to solve this problem.

\subsection{Coding and evolution operations}

In this study, the adopted method of chromosome coding, the crossover operator and the mutation operator were referred to the literature of Liu et al. [17].

\subsection{Fast non-dominant sort}

The core of solving multi-objective optimization problem is to find Pareto optimal solution sets [7]. Performing the fast non-dominated sorting refers to hierarchically ranking based on the advantages and disadvantages of all individuals in the population. If the individuals are in the same layer, the individuals are dominated by no others, while in different layers, the individuals are either dominated by others or dominate others. The specific operations are as follows.

We assume a variable $n_{i}$ and set $S_{i}$ for each individual $i$ of the population $Z$, where $n_{i}$ is the number of individuals dominating the individual $i$ and $S_{i}$ is the set of individuals dominated by the individual $i$ in the population. Let $l=1, Z^{\prime}=Z$;

(1) To calculate the $n_{i}$ and $S_{i}$ of each individual $i$ in the population $Z$;

(2) To find out the individuals of $n_{i}=0$ in the population $Z^{\prime}$, and put them into the current set $P_{l}$ ( $P_{l}$ represents the $l$ layer of the set), and execute $Z^{\prime}=Z^{\prime} \backslash P_{l}$;

(3) To perform $n_{j}=n_{j}-1$ on each individual $j$ in the set $S_{i}$ which corresponds to each individual $i$ in the current set $P_{l}$;

(4) If the set $Z^{\prime}$ is not empty, $l=l+1$, and turn to step (2). Otherwise, terminate the algorithm.

Finally, the individuals of the population $Z$ are divided into $l$ layers. 


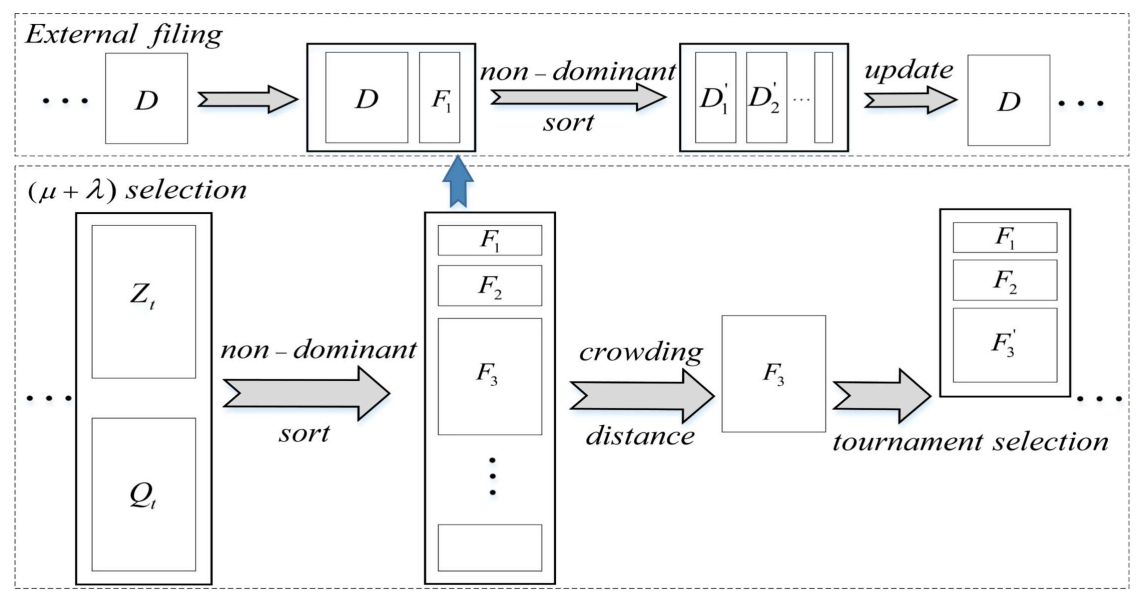

FIGURE 2. Integrating the $(\mu+\lambda)$ selection method elite retention and the external filing elite retention.

\subsection{Crowding distance}

The crowding distance is applied to measure the degree of similarity between individuals in the population. The closer the crowding distance of the individuals, the more similar to each other. Assume the number of the objective function is $m$ in the constructed model. Individuals are ranked in ascending order based on the $k$ th objective value of all individuals in the population. Besides, $f_{k}^{i+1}$ and $f_{k}^{i-1}$ represent the function values of two individuals adjacent to the individual $i$ on the $k$ th objective. The $f_{k}^{\max }$ and $f_{k}^{\min }$ are the maximum and minimum values. The crowding distance $d_{i}$ of an individual $i$ is calculated as follows:

$$
d_{i}= \begin{cases}\infty, & \text { ranked in the first and last individuals } \\ \sum_{k=1}^{m} \frac{f_{k}^{i+1}-f_{k}^{i-1}}{f_{k}^{\max }-f_{k}^{\min }}, & \text { otherwise. }\end{cases}
$$

\subsection{Tournament selection}

Tournament selection is random sampling with replacement used to select parents in producing offspring. Firstly, several individuals are randomly taken out of the population, and then the individuals with larger crowding distance are selected and retained to the next generation population set. Secondly, repeat the operation until as many individuals as the next generation of the population need to be saved. The purpose of this operation is to maintain population diversity.

\subsection{Elitism strategy}

In the multi-objective optimization algorithm, the elitism strategy can effectively prevent the loss of excellent individuals in the evolutionary process. The $(\mu+\lambda)$ selection [10] method (strategy I) can preserve excellent genes and make the population evolve in a better direction. However, with the operation of crossover and mutation, it may lead to the failure of excellent individuals to continue to the last generation of the population. The external filing method can effectively avoid this problem and make the elite individuals preserved in the evolution process. Therefore, the second strategy (strategy II) of this study combines the $(\mu+\lambda)$ selection method with the external filing method to achieve elite retention. The following is a schematic diagram of the NSGA-II elite retention process, as shown in the Figure 2.

The specific flow expression of NSGA-II is as follows:

Step 1. To generate an initial population $Z_{t}$ with size $\mu$ and let iterations times as $\Upsilon$, crossover probability as $P_{c}$ and mutation probability as $P_{m}$; 
Step 2. To perform the crossover and mutation operation on the population $Z_{t}$ to generate a new offspring population $Q_{t}$, and then combine $Z_{t}$ with $Q_{t}$ to form a population $R_{t}$ with size $(\mu+\lambda)$;

Step 3. To evaluate the bi-objective fitness for each individual in the population $R_{t}$, and perform fast nondominated sorting;

Step 4. To adopt the external filing method to update the external archive sets;

Step 5. To apply the crowding distance and tournament selection methods to generate an offspring population $Z_{t+1}$

Step 6. If the iteration termination condition is not satisfied, turn to step (2). Otherwise, terminate the algorithm.

\section{Results And Discussion}

\subsection{Analysis of algorithms}

To compare the performance of the strategy I and strategy II, we employ three evaluation metrics, including the diversity, the convergence and the distribution degree of the Pareto Frontier solution sets. Set $A$ and $B$ as two different Pareto Frontier solution sets. The evaluation index of diversity depends on the number of solutions in solution Pareto Frontier set. Assuming $u$ and $v$ are two solutions in the Pareto Frontier solution set. Equation (4.1) means the dominance between $u$ and $v$. Equation (4.2) represents the relative convergence rate of solution set $A$ to $B$ [40]. $r(A, B)>r(B, A)$ indicates that $A$ has better convergence.

$$
\begin{aligned}
s_{(u, v)} & = \begin{cases}1, & \text { if } u \text { dominates } v \\
0, & \text { if } v \text { dominates } u\end{cases} \\
r(A, B) & =\frac{\sum_{u \in A} \sum_{\in B} s_{(u, v)}}{\sum_{u \in A} \sum_{v \in B} s_{(u, v)}+\sum_{u \in B} \sum_{v \in A} s_{(u, v)}} .
\end{aligned}
$$

Equation (4.3) represents that the function of $F(A)$ is to evaluate the distribution degree of solutions in the Pareto Frontier solution set $A$ [24], where $d_{i}^{*}=\min _{j \in A \backslash\{j \neq i\}} \sum_{k=1}^{m}\left|f_{k}^{i}-f_{k}^{j}\right|, \bar{d}=\left(\sum_{i=1}^{\|A\|} d_{i}^{*}\right) /\|A\|, i, j \in A, \alpha$ is a constant and the number of the objective functions is $m$. The more uniform the solution distribution in the solution set $A$, the value of the $F(A)$ is bigger.

$$
F(A)=\alpha\left[\sqrt{\frac{1}{\|A\|-1} \sum_{i=1}^{\|A\|}\left(d_{i}^{*}-\bar{d}\right)^{2}}\right]^{-1} .
$$

In Solomon C101 case, we randomly select 20 nodes, 40 nodes and 60 nodes to construct three multimodal transport networks respectively. In the three networks, transportation duration, cost and modes are generated randomly. The data of transfer are shown in Table 3. The transfer nodes have a fixed timetable for each mode of transportation. The timetable of railway transportation is $3: 00 ; 6: 00 ; 9: 00 ; 12: 00 ; 15: 00 ; 18: 00 ; 21: 00$. The timetable of waterway transportation is 11:00; 18:00. Highway transportation has no timetable. The interval robust control parameter corresponding to all edges in transportation network is set to 1 . It is worth noting that algorithm program is conducted using MATLAB2014b software on an Intel Corei5 PC with 4 gigabytes of RAM and over $2.5 \mathrm{GHz}$ CPU.

Taking the 40 nodes network as an example, Figure 3 depicts the average total duration and cost of all individuals in each generation of the population during the evolution of NSGA-II. Meanwhile, it shows that the objectives of total duration and cost are searched towards the smallest direction by NSGA-II and verifies the reasonableness of the designed algorithm. Figure 4 illustrates the trend of changes in the obtained minimum total duration and cost through both strategy I and strategy II, respectively, as the population evolves. According to 


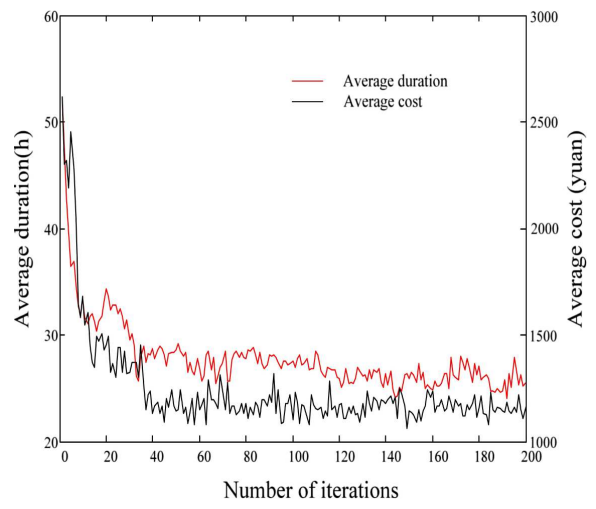

Figure 3. The variation of average cost and duration.

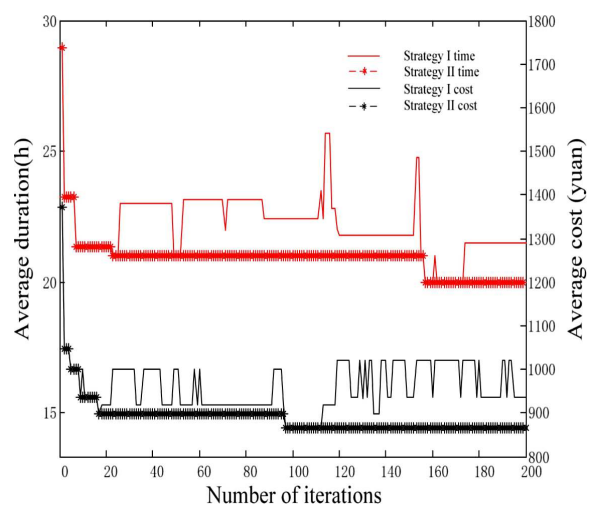

Figure 4. The variation of the minimum duration and cost under comparison strategies.

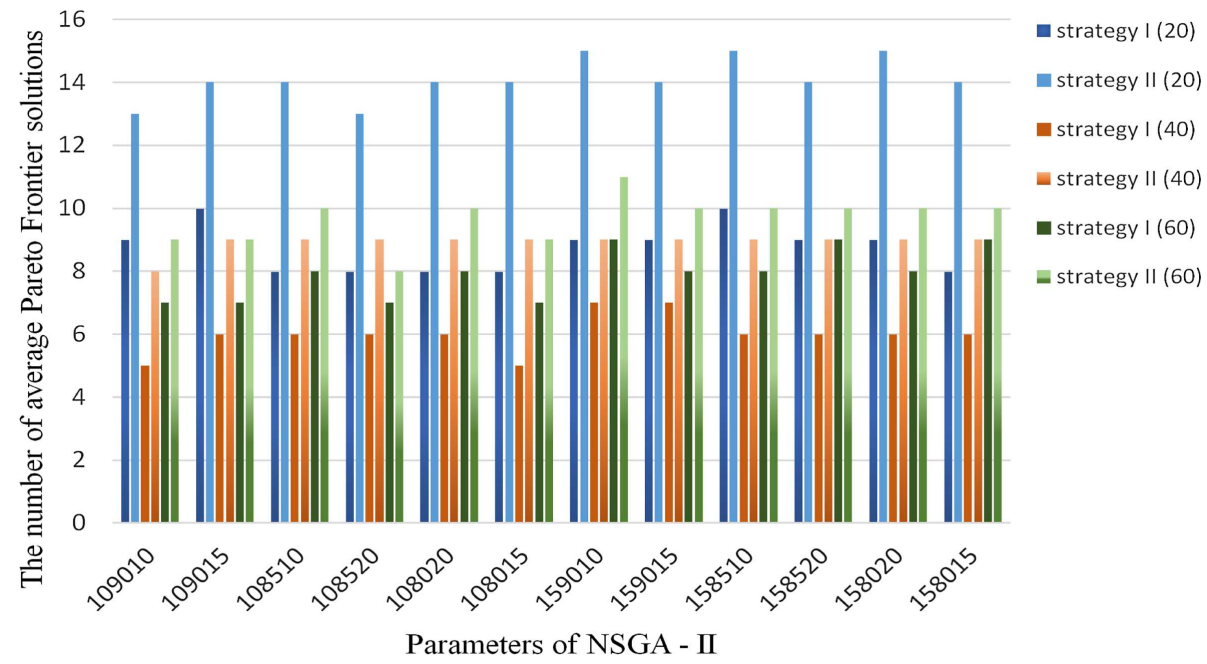

Figure 5. Average number of Pareto Frontier solutions under different strategies and networks. 


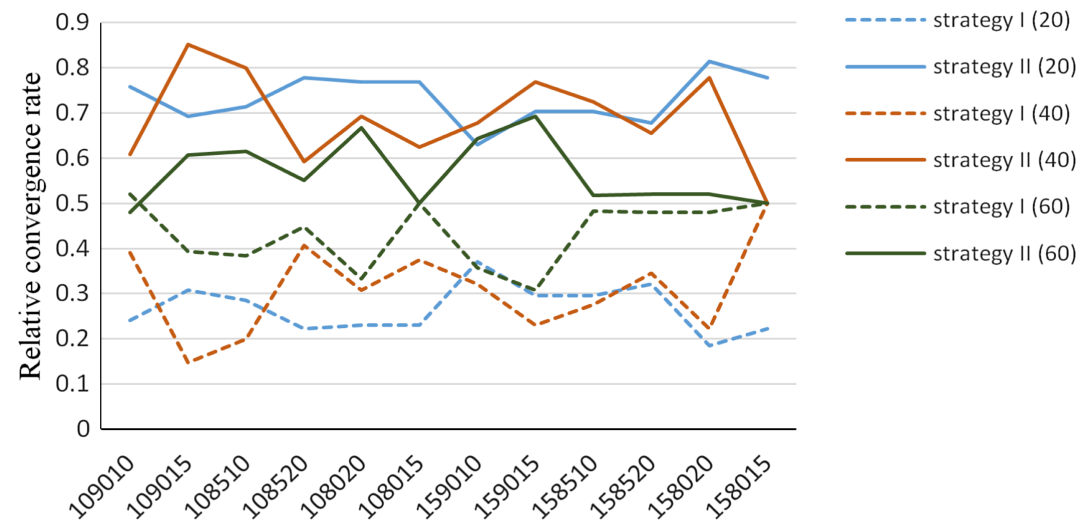

Parameters of NSGA - II

FIGURE 6. Relative convergence rate under different solution strategies and networks.

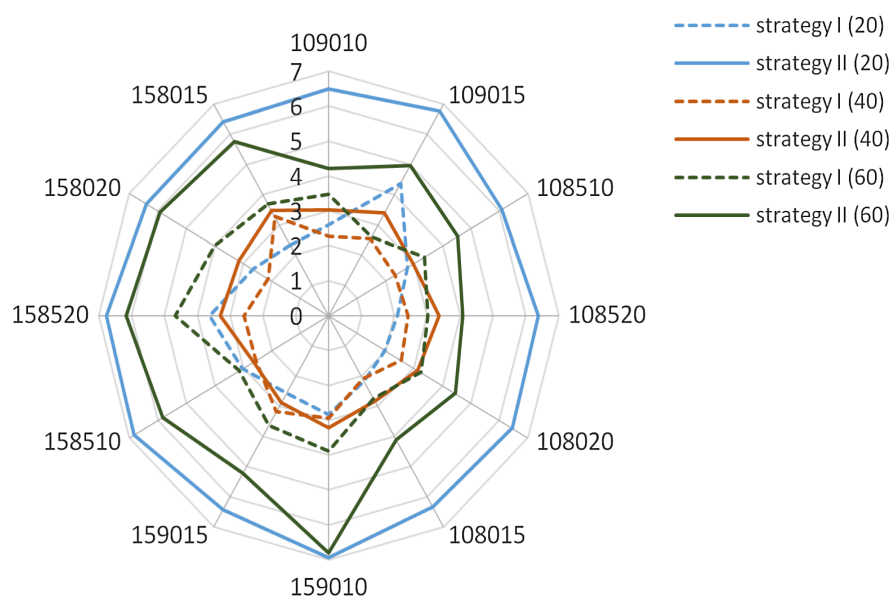

Figure 7. The distribution of the solutions under different solution strategies and networks.

Figure 4, the minimum value of each objective obtained through Strategy I will fluctuate, leading to the loss of the elite. By contrast, strategy II can better achieve elite retention.

Different NSGA-II parameters are set (In the "109010", the first and second digits represent a population size of $100(10 * 10)$, the third and fourth digits represent a crossover probability of $0.90(90 / 100)$ and the fifth and sixth digits represent a mutation probability of $0.10(10 / 100)$ ) and iterations are performed 200 times. In Figures 5-7 we graphically describe the diversity, the convergence and the distribution degree of the Pareto Frontier solution sets obtained by adopting strategy I and strategy II under different network scales after the algorithm runs 30 times. In Figure 7, the axis index represents the distribution degree of Pareto optimal solution set, and the strategy II has more desirable ability to make the dispersion of solution more uniform under different genetic parameter combinations. The results of the three algorithm evaluation indicators can be significantly verify that Strategy II is superior to strategy I.

Different parameters are set (The "2109010" is composed of "2" and "109010", the "2" represents that the number of network nodes is $2 * 10$ and the "109010" as explained above) and iterations are performed 200 times. Considered different parameters and strategies, Table 2 states the mean deviation of the minimum and 
TABLE 2. The evaluation metric data of the two solution strategies.

\begin{tabular}{|c|c|c|c|c|c|c|c|c|c|c|}
\hline \multirow{3}{*}{ Param } & \multicolumn{5}{|c|}{ Strategy I } & \multicolumn{5}{|c|}{ Strategy II } \\
\hline & \multicolumn{2}{|c|}{ Obj.1 } & \multicolumn{2}{|c|}{ Obj.2 } & \multirow[b]{2}{*}{$\mathrm{CPU}(\mathrm{s})$} & \multicolumn{2}{|c|}{ Obj.1 } & \multicolumn{2}{|c|}{ Obj.2 } & \multirow[b]{2}{*}{$\mathrm{CPU}(\mathrm{s})$} \\
\hline & $\begin{array}{l}\text { Ave. } \\
\text { min } \%\end{array}$ & $\begin{array}{l}\text { Ave. } \\
\max \%\end{array}$ & $\begin{array}{l}\text { Ave. } \\
\text { min\% }\end{array}$ & $\begin{array}{l}\text { Ave. } \\
\max \%\end{array}$ & & $\begin{array}{l}\text { Ave. } \\
\text { min } \%\end{array}$ & $\begin{array}{l}\text { Ave. } \\
\max \%\end{array}$ & $\begin{array}{l}\text { Ave. } \\
\text { min } \%\end{array}$ & $\begin{array}{l}\text { Ave. } \\
\max \%\end{array}$ & \\
\hline 2109010 & 10.54 & 9.49 & 8.47 & 7.25 & 69.02 & 2.45 & 1.76 & 0.94 & 2.31 & 73.36 \\
\hline 2109015 & 5.92 & 8.45 & 8.51 & 3.98 & 70.78 & 1.99 & 1.53 & 0.62 & 2.29 & 73.67 \\
\hline 2108510 & 11.15 & 8.65 & 6.71 & 10.22 & 70.38 & 1.51 & 1.77 & 0.76 & 2.15 & 74.13 \\
\hline 2108520 & 10.61 & 10.45 & 6.29 & 7.35 & 71.32 & 1.04 & 2.46 & 1.17 & 1.94 & 71.43 \\
\hline 2108020 & 10.95 & 12.49 & 6.48 & 4.54 & 71.56 & 1.28 & 2.07 & 0.69 & 1.88 & 72.72 \\
\hline 2108015 & 11.31 & 10.38 & 8.49 & 8.18 & 72.14 & 1.69 & 1.81 & 1.01 & 2.15 & 73.73 \\
\hline 2159010 & 6.64 & 9.69 & 8.73 & 5.03 & 133.02 & 1.30 & 2.28 & 0.84 & 1.66 & 134.95 \\
\hline 2159015 & 5.88 & 7.84 & 8.56 & 5.56 & 137.07 & 0.44 & 1.88 & 0.78 & 1.18 & 136.30 \\
\hline 2158510 & 6.94 & 9.60 & 7.45 & 3.97 & 137.49 & 0.80 & 1.88 & 0.84 & 0.87 & 140.16 \\
\hline 2158520 & 8.60 & 8.13 & 5.01 & 7.12 & 129.60 & 1.06 & 2.02 & 1.15 & 1.62 & 132.00 \\
\hline 2158020 & 6.36 & 9.13 & 6.80 & 5.40 & 137.60 & 0.93 & 1.96 & 0.44 & 1.77 & 138.65 \\
\hline 2158015 & 12.19 & 8.37 & 6.44 & 7.44 & 135.80 & 1.43 & 1.68 & 0.65 & 2.10 & 136.43 \\
\hline 4109010 & 10.60 & 5.19 & 3.26 & 27.22 & 78.67 & 4.14 & 2.62 & 1.62 & 17.35 & 81.69 \\
\hline 4109015 & 10.44 & 5.33 & 2.73 & 21.54 & 81.80 & 4.83 & 1.84 & 2.05 & 26.29 & 83.44 \\
\hline 4108510 & 11.98 & 7.36 & 4.31 & 24.99 & 84.85 & 5.16 & 1.40 & 0.58 & 15.97 & 85.60 \\
\hline 4108520 & 12.33 & 7.77 & 3.56 & 23.97 & 75.60 & 3.88 & 2.91 & 2.29 & 14.31 & 80.23 \\
\hline 4108020 & 9.21 & 4.03 & 2.03 & 35.68 & 79.62 & 6.05 & 1.03 & 2.25 & 21.74 & 83.30 \\
\hline 4108015 & 6.63 & 5.37 & 3.04 & 26.12 & 83.40 & 5.06 & 1.78 & 0.47 & 20.93 & 82.18 \\
\hline 4159010 & 8.82 & 5.01 & 2.59 & 26.24 & 142.36 & 5.83 & 1.06 & 1.46 & 17.89 & 143.39 \\
\hline 4159015 & 5.54 & 8.68 & 5.40 & 26.23 & 145.44 & 5.44 & 1.33 & 2.19 & 14.43 & 147.71 \\
\hline 4158510 & 7.78 & 5.03 & 3.31 & 34.04 & 146.35 & 5.61 & 1.11 & 1.97 & 14.83 & 150.07 \\
\hline 4158520 & 9.34 & 4.53 & 2.28 & 31.04 & 137.58 & 5.10 & 1.34 & 1.41 & 24.92 & 140.22 \\
\hline 4158020 & 7.91 & 5.51 & 3.28 & 31.69 & 146.45 & 5.84 & 0.95 & 1.57 & 15.46 & 151.25 \\
\hline 4158015 & 9.55 & 4.29 & 1.43 & 33.48 & 147.33 & 5.79 & 0.50 & 1.62 & 26.83 & 149.67 \\
\hline 6109010 & 12.15 & 7.95 & 3.93 & 16.67 & 87.78 & 4.67 & 5.70 & 2.91 & 8.56 & 88.07 \\
\hline 6109015 & 4.84 & 6.06 & 2.97 & 11.92 & 86.72 & 5.33 & 5.70 & 1.41 & 9.25 & 90.24 \\
\hline 6108510 & 4.94 & 8.66 & 3.77 & 9.95 & 88.45 & 4.18 & 5.91 & 0.91 & 6.33 & 92.50 \\
\hline 6108520 & 7.4 & 8.35 & 5.28 & 13 & 85.51 & 5.41 & 5.48 & 1.65 & 9.17 & 86.69 \\
\hline 6108020 & 4.61 & 8.22 & 3.31 & 6.88 & 91.93 & 3.74 & 6.76 & 1.53 & 4.25 & 94.47 \\
\hline 6108015 & 8.18 & 9.42 & 4.24 & 14.48 & 86.2 & 5.56 & 5.22 & 1.29 & 9.93 & 88.89 \\
\hline 6159010 & 3.03 & 5.99 & 2.59 & 4.5 & 150.69 & 2.09 & 5.36 & 1.29 & 3.80 & 151.23 \\
\hline 6159015 & 4.52 & 8.09 & 4.99 & 6.93 & 153.22 & 3.32 & 5.63 & 0.94 & 4.55 & 152.31 \\
\hline 6158510 & 2.93 & 6.61 & 2.91 & 4.84 & 154.64 & 2.12 & 5.49 & 0.63 & 2.58 & 158.58 \\
\hline 6158520 & 5.33 & 5.5 & 2.51 & 7.32 & 148.62 & 2.77 & 4.67 & 1.15 & 2.43 & 149.58 \\
\hline 6158020 & 5.01 & 8.63 & 3.32 & 9.32 & 156.54 & 2.26 & 5.12 & 0.91 & 4.69 & 159.22 \\
\hline 6158015 & 4.04 & 8.47 & 3.57 & 7.44 & 151.99 & 3.1 & 5.7 & 0.95 & 6.42 & 152.24 \\
\hline
\end{tabular}

maximum of each objective value and the average solution time CPU(s) after the algorithm was run 30 times. From the results presented here, it is obvious that the average deviation of each objective value obtained by strategy II is relatively small under the same parameters and the fluctuation of the average deviation is also relatively small when changed NSGA-II parameters. The CPU is mainly related to the network scale and the population size, yet it is no meaningful relationship with the strategy selection. The analysis shows that strategy II has a desirable performance of stability in solving multi-objective multimodal transportation optimization problems under uncertainty. 


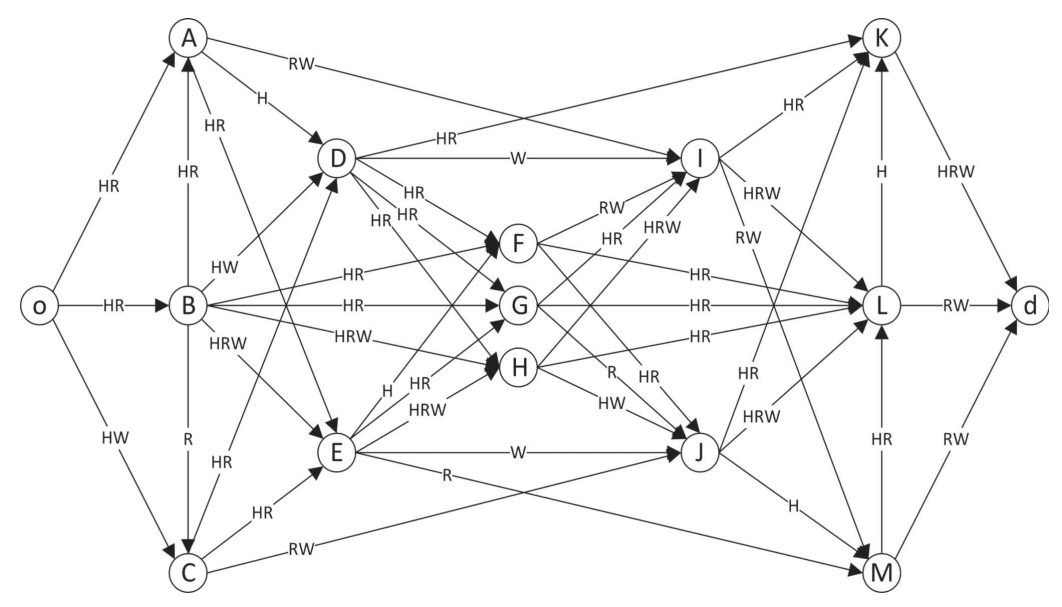

FiguRE 8. Multimodal transportation network.

TABle 3. Parameters of transfer.

\begin{tabular}{lllllll}
\hline \hline & \multicolumn{2}{l}{$\begin{array}{l}\text { Unit transferred duration } \\
\text { between different mode of } \\
\text { transportation }(\mathrm{h})\end{array}$} & \multicolumn{4}{l}{$\begin{array}{l}\text { Unit transferred cost between } \\
\text { different modes of transportation } \\
\text { (yuan) }\end{array}$} \\
\cline { 2 - 7 } & Highway & Railway & Waterway & Highway & Railway & Waterway \\
\hline Highway & 1 & 1.5 & 2 & 30 & 30 & 40 \\
Railway & 1.5 & 2 & 4 & 30 & 40 & 50 \\
Waterway & 2 & 4 & 3 & 40 & 50 & 50 \\
\hline
\end{tabular}

\subsection{Problem instances}

We assume, for simplicity, there are 100 tons of goods need to be transported from node $o$ to node $d$ through multimodal transportation. The structure of the multimodal transportation network is shown in Figure 8 . The transfer data are shown in Table 3, Table 4 presents the data on the multimodal transportation network. Nodes $A$ to $M$ have a fixed timetable for each mode of transportation. The railway timetable is: $3: 00 ; 6: 00 ; 9: 00 ; 12: 00$; 15:00; 18:00; 21:00. The waterway timetable is: 11:00; 18:00. The highway has no timetable. The departure time of the goods at the origin node is 7:30 am. The interval robust control parameters corresponding to all edges of highway, railway and waterway transportation duration are all set as 1 , which indicates that the decision makers are pessimistic about the uncertain transportation duration. It is required to determine a reasonable route scheme to minimize the total duration and cost of multimodal transportation.

In this numerical instance, the population size is 100 , the number of iterations is 200 , Cross probability is 0.8 and mutation probability is 0.2 of the NSGA-II, and two different elite strategies (strategy I and strategy II) are employed. $\Re_{1}$ means Pareto Frontier solution set obtained by strategy I, which contains 8 solutions, $\Re_{2}$ means Pareto Frontier solution set obtained by strategy II, which contains 12 solutions. The distribution and convergence of its solution sets can be understood from Figure 9. In addition, it further reveals that there are five solutions which have the same objective function value in $\Re_{1}$ and $\Re_{2}$, and three of the solutions in $\Re_{1}$ are dominated by some of the solutions in $\Re_{2}$.

In the solved instances, 12 groups of non-dominant schemes have been obtained through strategy II as shown in Table 5, but there is no absolute advantage of each group scheme in transportation duration or cost. The results can be referenced for decision makers. 
TABLE 4. Multimodal transportation network data.

Point pair; highway data; railway data; waterway data (data format: [transportation duration interval number (h)], cost (yuan/100TUD). $\sim$ means empty)

\begin{tabular}{|c|c|}
\hline $\mathrm{EJ} ; \sim ; \sim ;[67,78], 442$ & $\mathrm{EM} ; \sim ;[59,70], 618 ; \sim$ \\
\hline $\mathrm{JM} ;[17,22], 2213 ; \sim ; \sim$ & LK; $[17,20], 1206 ; \sim ; \sim$ \\
\hline $\mathrm{AD} ;[27,32], 1663 ; \sim ; \sim$ & $\mathrm{EF} ;[21,26], 571 ; \sim ; \sim$ \\
\hline $\mathrm{BC} ; \sim ;[85,96], 611 ; \sim$ & GJ; $\sim ;[65,77], 1078 ; \sim$ \\
\hline $\mathrm{DI} ; \sim ; \sim ;[89,107], 463$ & $\mathrm{Md} ; \sim ;[40,46], 1046 ;[104,115], 465$ \\
\hline $\mathrm{oA} ;[15,24], 971 ;[41,48], 674 ; \sim$ & $\mathrm{oB} ;[16,21], 1100 ;[41,45], 653 ; \sim$ \\
\hline $\mathrm{AE} ;[18,25], 1037 ;[44,52], 475 ; \sim$ & $\mathrm{AI} ; \sim ;[34,40], 913 ;[116,120], 367$ \\
\hline $\mathrm{BD} ;[26,40], 1004 ; \sim ;[92,103], 497$ & $\mathrm{BF} ;[36,40], 1527 ;[63,67], 767 ; \sim$ \\
\hline $\mathrm{CD} ;[18,30], 1890 ;[54,64], 653 ; \sim$ & $\mathrm{CE} ;[12,23], 923 ;[59,65], 681 ; \sim$ \\
\hline DF; [41,44], 1158; [36,43], 663; & DG; $[37,41], 1193 ;[54,65], 460 ; \sim$ \\
\hline DK; $[43,46], 1397 ;[39,48], 528 ; \sim$ & EG; [26,37], 1900; [51,58], 472; \\
\hline FJ; $[17,28], 1592 ;[35,45], 739 ; \sim$ & FL; $[19,24], 1784 ;[45,57], 786 ; \sim$ \\
\hline GL; $[32,35], 1825 ;[38,46], 649 ; \sim$ & HJ; [33,45], 1818; ; [100,104], 409 \\
\hline IK; $[9,21], 1817 ;[44,48], 459 ; \sim$ & $\mathrm{IM} ; \sim ;[33,45], 1098 ;[95,101], 367$ \\
\hline $\mathrm{Ld} ; \sim ;[40,52], 1053 ;[69,73], 468$ & ML; $[40,46], 1119 ;[48,54], 516 ; \sim$ \\
\hline oC; [16,22], 1887; ; [100,111], 364 & FI; ; [44,53], 888; [103,113], 466 \\
\hline $\mathrm{BA} ;[35,43], 1029 ;[48,57], 782 ; \sim$ & GI; $[41,48], 1803 ;[48,59], 671 ; \sim$ \\
\hline BG; [37,47], 1350; [37,43], 423; & HL; $[16,25], 1608 ;[50,62], 716 ; \sim$ \\
\hline CJ; ; [30,42], 1203; [85,97], 361 & $\mathrm{JK} ;[44,48], 1233 ;[55,63], 704 ; \sim$ \\
\hline $\mathrm{DH} ;[39,45], 1026 ;[38,46], 749 ; \sim$ & $\mathrm{EH} ;[36,41], 1559 ;[54,57], 855 ;[112,115], 439$ \\
\hline $\mathrm{BE} ;[35,39], 1921 ;[46,55], 629 ;[64,76], 408$ & $\mathrm{BH} ;[42,47], 1388 ;[37,41], 981 ;[65,74], 512$ \\
\hline IL; $[26,37], 1386 ;[43,51], 660 ;[66,76], 403$ & $\mathrm{Kd} ;[11,20], 1557 ;[50,61], 879 ;[83,86], 528$ \\
\hline HI; [23,32], 1909; [43,53], 624; [93,99], 522 & $\mathrm{JL} ;[27,35], 1140 ;[43,48], 728 ;[72,78], 397$ \\
\hline
\end{tabular}

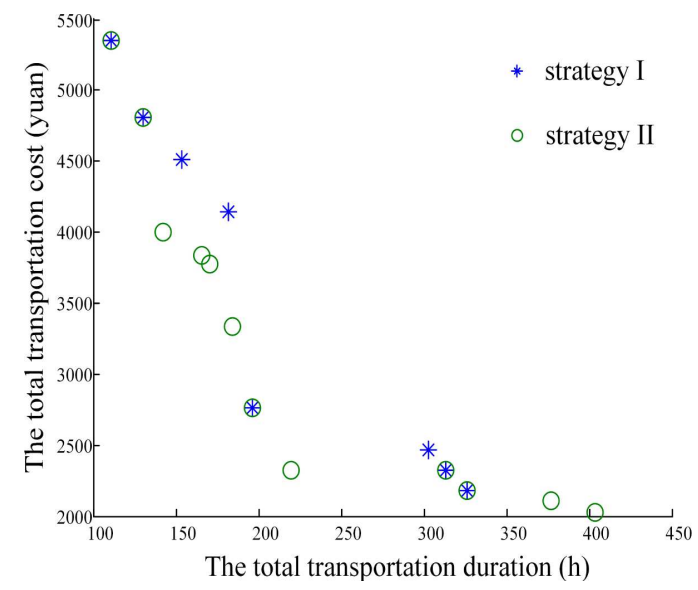

Figure 9. Distribution and convergence of Pareto Frontier solution sets under different solution strategies. 
TABLE 5. Results of problem instance by solution strategy II.

\begin{tabular}{|c|c|c|c|}
\hline & Route and mode of transportation & Total duration $(\mathrm{h})$ & Total cost (yuan) \\
\hline Scheme 1 & $\mathrm{o}(\mathrm{H}) \mathrm{A}(\mathrm{R}) \mathrm{I}(\mathrm{H}) \mathrm{K}(\mathrm{H}) \mathrm{d}$ & 110 & 5348 \\
\hline Scheme 2 & $\mathrm{o}(\mathrm{H}) \mathrm{A}(\mathrm{H}) \mathrm{D}(\mathrm{R}) \mathrm{K}(\mathrm{H}) \mathrm{d}$ & 130 & 4809 \\
\hline Scheme 3 & $\mathrm{o}(\mathrm{H}) \mathrm{A}(\mathrm{R}) \mathrm{I}(\mathrm{R}) \mathrm{K}(\mathrm{H}) \mathrm{d}$ & 142 & 4000 \\
\hline Scheme 4 & $\mathrm{o}(\mathrm{R}) \mathrm{B}(\mathrm{H}) \mathrm{D}(\mathrm{R}) \mathrm{K}(\mathrm{H}) \mathrm{d}$ & 166 & 3832 \\
\hline Scheme 5 & $\mathrm{o}(\mathrm{H}) \mathrm{A}(\mathrm{H}) \mathrm{E}(\mathrm{R}) \mathrm{M}(\mathrm{R}) \mathrm{d}$ & 170 & 3772 \\
\hline Scheme 6 & $\mathrm{o}(\mathrm{H}) \mathrm{A}(\mathrm{R}) \mathrm{I}(\mathrm{R}) \mathrm{K}(\mathrm{R}) \mathrm{d}$ & 184 & 3332 \\
\hline Scheme 7 & $\mathrm{o}(\mathrm{H}) \mathrm{B}(\mathrm{R}) \mathrm{G}(\mathrm{R}) \mathrm{L}(\mathrm{W}) \mathrm{d}$ & 196 & 2760 \\
\hline Scheme 8 & $\mathrm{o}(\mathrm{R}) \mathrm{B}(\mathrm{R}) \mathrm{G}(\mathrm{R}) \mathrm{L}(\mathrm{W}) \mathrm{d}$ & 220 & 2323 \\
\hline Scheme 9 & $\mathrm{o}(\mathrm{R}) \mathrm{A}(\mathrm{W}) \mathrm{I}(\mathrm{R}) \mathrm{L}(\mathrm{W}) \mathrm{d}$ & 313 & 2319 \\
\hline Scheme 10 & $\mathrm{o}(\mathrm{R}) \mathrm{A}(\mathrm{W}) \mathrm{I}(\mathrm{R}) \mathrm{K}(\mathrm{W}) \mathrm{d}$ & 326 & 2178 \\
\hline Scheme 11 & $\mathrm{o}(\mathrm{W}) \mathrm{C}(\mathrm{W}) \mathrm{J}(\mathrm{R}) \mathrm{K}(\mathrm{W}) \mathrm{d}$ & 377 & 2107 \\
\hline Scheme 12 & $\mathrm{o}(\mathrm{R}) \mathrm{A}(\mathrm{W}) \mathrm{I}(\mathrm{W}) \mathrm{M}(\mathrm{W}) \mathrm{d}$ & 403 & 2023 \\
\hline
\end{tabular}

TABLE 6 . Results of problem instance by solution strategy II when $\hbar_{i, j}^{1}=1, \hbar_{i, j}^{2}=0.5$ and $\hbar_{i, j}^{3}=0.8$.

\begin{tabular}{|c|c|c|c|}
\hline & Route and mode of transportation & Total duration $(\mathrm{h})$ & Total cost (yuan) \\
\hline Scheme 1 & $\mathrm{o}(\mathrm{H}) \mathrm{A}(\mathrm{R}) \mathrm{I}(\mathrm{H}) \mathrm{K}(\mathrm{H}) \mathrm{d}$ & 108 & 5348 \\
\hline Scheme 2 & $\mathrm{o}(\mathrm{H}) \mathrm{A}(\mathrm{H}) \mathrm{D}(\mathrm{R}) \mathrm{K}(\mathrm{H}) \mathrm{d}$ & 126 & 4809 \\
\hline Scheme 3 & $\mathbf{o}(\mathbf{H}) \mathbf{B}(\mathbf{H}) \mathbf{D}(\mathbf{R}) \mathbf{K}(\mathbf{H}) \mathrm{d}$ & 138 & 4279 \\
\hline Scheme 4 & $\mathrm{o}(\mathrm{H}) \mathrm{A}(\mathrm{R}) \mathrm{I}(\mathrm{R}) \mathrm{K}(\mathrm{H}) \mathrm{d}$ & 140 & 4000 \\
\hline Scheme 5 & $\mathrm{o}(\mathrm{H}) \mathrm{A}(\mathrm{H}) \mathrm{E}(\mathrm{R}) \mathrm{M}(\mathrm{R}) \mathrm{d}$ & 164 & 3772 \\
\hline Scheme 6 & $\mathbf{o}(\mathbf{R}) \mathbf{A}(\mathbf{R}) \mathbf{I}(\mathbf{R}) \mathbf{K}(\mathbf{H}) \mathbf{d}$ & 164 & 3713 \\
\hline Scheme 7 & $\mathbf{o}(\mathbf{H}) \mathrm{B}(\mathbf{R}) \mathrm{G}(\mathbf{R}) \mathbf{L}(\mathbf{R}) \mathrm{d}$ & 167 & 3335 \\
\hline Scheme 8 & $\mathrm{o}(\mathrm{H}) \mathrm{A}(\mathrm{R}) \mathrm{I}(\mathrm{R}) \mathrm{K}(\mathrm{R}) \mathrm{d}$ & 176 & 3332 \\
\hline Scheme 9 & $\mathbf{o}(\mathbf{H}) \mathbf{A}(\mathbf{R}) \mathbf{E}(\mathbf{R}) \mathbf{M}(\mathbf{R}) \mathrm{d}$ & 190 & 3220 \\
\hline Scheme 10 & $\mathrm{o}(\mathrm{H}) \mathrm{B}(\mathrm{R}) \mathrm{G}(\mathrm{R}) \mathrm{L}(\mathrm{W}) \mathrm{d}$ & 193 & 2760 \\
\hline Scheme 11 & $\mathrm{o}(\mathrm{R}) \mathrm{B}(\mathrm{R}) \mathrm{G}(\mathrm{R}) \mathrm{L}(\mathrm{W}) \mathrm{d}$ & 217 & 2323 \\
\hline Scheme 12 & $\mathrm{o}(\mathrm{R}) \mathrm{A}(\mathrm{W}) \mathrm{I}(\mathrm{R}) \mathrm{L}(\mathrm{W}) \mathrm{d}$ & 298 & 2319 \\
\hline Scheme 13 & $\mathbf{o}(\mathbf{R}) \mathbf{A}(\mathbf{W}) \mathbf{I}(\mathbf{W}) \mathbf{L}(\mathbf{W}) \mathbf{d}$ & 322 & 2062 \\
\hline Scheme 14 & $\mathbf{o}(\mathbf{W}) \mathbf{C}(\mathbf{W}) \mathbf{J}(\mathbf{W}) \mathbf{L}(\mathbf{W}) \mathbf{d}$ & 385 & 1740 \\
\hline
\end{tabular}

Notes. The bold values are new ob-tained solutions after adjusting the robust parameters.

Robust control parameters can be adjusted to reflect the risk preferences of different decision makers. For example, $\hbar_{i, j}^{1}=1$ and $\hbar_{i, j}^{2}=0.5$ indicate as pessimistic about the transportation duration for highway and optimistic for railway and $\hbar_{i, j}^{3}=0.8$ means the degree of risk decision preferences for waterway duration between pessimistic and optimistic. The scheme calculation results are shown in Table 6. Compared with Table 5, it can be seen that pareto solution set is variable under different robust control parameters.

\section{Conclusions}

In this paper, we establish a multi-objective multimodal transportation route robust optimization model with the minimum transportation duration and cost based on interval number under the uncertainty of transportation duration. NSGA-II with the elitist retention strategy is designed to solve the problem. High-quality solutions are obtained in numerical simulation, which confirms that the algorithm is effective in solving the bi-objective 
optimization problem. In the algorithm analysis, we propose a feasible method to evaluate the performance of the algorithm and the qualities of Pareto Frontier solution sets. The analysis indicates that adding the external archive strategy can improve the performance of the algorithm and the quality of the solutions. Decision makers can adjust robust control parameters to reflect their risk preference for uncertain transportation duration, and enable to balance the duration and cost interests based on the schemes. Finally, in future research, more efficient algorithms could be designed for the proposed problem in this paper to further improve the quality of the solutions.

Acknowledgements. The present research work has been supported by MOE (Ministry of Education in China) Project of Humanities and Social Sciences [No. 17YJA630079] and Social Science Planning Project of Chongqing, China [No. 2019YBGL049]. The authors gratefully acknowledge the support of these institutions.

\section{REFERENCES}

[1] A. Abbassi, E.H.A. Ahmed and J. Boukachour, Robust optimisation of the intermodal freight transport problem: modeling and solving with an efficient hybrid approach. J. Comput. Sci. 30 (2019) 127-142.

[2] G. Assadipour, G.Y. Ke and M. Verma, Planning and managing intermodal transportation of hazardous materials with capacity selection and congestion. Transp. Res. Part E: Logistics Transp. Rev. 76 (2015) 45-57.

[3] H. Ayed, Z. Habbas, D. Khadraoui and F. Galvez, A parallel algorithm for solving time dependent multimodal transport problem [C]. In: International IEEE Conference on Intelligent Transportation Systems. IEEE, Washington, DC (2011).

[4] F. Bruns and S. Knust, Optimized load planning of trains in intermodal transportation. OR Spectrum 34 (2012) $511-533$.

[5] T.S. Chang, Best routes selection in international intermodal networks. J. Dalian Maritime Univ. 35 (2008) $2877-2891$.

[6] J.H. Cho, H.S. Kim, H.R. Choi, N.K. Park and M.H. Kang, An intermodal transport network planning algorithm using dynamic programming. Appl. Intell. 36 (2012) 529-541.

[7] K. Deb, A. Pratap, S. Agarwal and T. Meyarivan, A fast and elitist multi-objective genetic algorithm: NSGA-II. IEEE Trans. Evol. Comput. 6 (2002) 182-197.

[8] E. Demir, W. Burgholzer, M. Hrušovský, E. Arıkan, W. Jammernegg and T.V. Woensel, A green intermodal service network design problem with travel time uncertainty. Transp. Res. Part B: Methodol. 93 (2016) 789-807.

[9] S. Fazayeli, A. Eydi and I.N. Kamalabadi, Location-routing problem in multimodal transportation network with time windows and fuzzy demands: presenting a two-part genetic algorithm. Comput. Ind. Eng. 119 (2018) 233-246.

[10] L.J. Fogel, A.J. Owens and M.J. Walsh, Artificial Intelligence Through Simulated Evolution. John Wiley \& Sons, New York, NY (1966).

[11] A. Ghaderi and R.L. Burdett, An integrated location and routing approach for transporting hazardous materials in a bi-modal transportation network. Transp. Res. Part E: Logistics Transp. Rev. 127 (2019) 49-65.

[12] S.E. Grasman, Dynamic approach to strategic and operational multimodal routing decisions. Int. J. Logistics Syst. Manage. 2 (2006) 96.

[13] C. Hao and Y. Yue, Optimization on combination of transport routes and modes on dynamic programming for a container multimodal transport system. Proc. Eng. 137 (2016) 382-390.

[14] M. Hrusovsky, E. Demir, W. Jammernegg and T.V. Woensel, Hybrid simulation and optimization approach for green intermodal transportation problem with travel time uncertainty. Flexible Serv. Manuf. J. 30 (2016) 486-516.

[15] J.S.L. Lam and Y. Gu, A market-oriented approach for intermodal network optimization meeting cost, time and environmental requirements. Int. J. Prod. Econ. 171 (2016) 266-274.

[16] L. Li, R.R. Negenborn and B. De Schutter, Intermodal freight transport planning - A receding horizon control approach. Transp. Res. Part C: Emerg. Technol. 60 (2015) 77-95.

[17] S. Liu, Y. Peng, Q.K. Song and Y.Y. Zhong, The robust shortest path problem for multimodal transportation considering timetable with interval data. Syst. Sci. Control Eng. 6 (2018) 68-78.

[18] C. Macharis and E. Pekin, Assessing policy measures for the stimulation of intermodal transport: a GIS-based policy analysis. J. Transp. Geogr. 17 (2009) 500-508.

[19] I.A. Martínez-Salazar, J. Molina, F. Ángel-Bello, T. Gómez and R. Caballero, Solving a bi-objective transportation location routing problem by metaheuristic algorithms. Eur. J. Oper. Res. 234 (2014) 25-36.

[20] G. Mavrotas and K. Florios, An improved version of the augmented $\epsilon$-constraint method (AUGMECON2) for finding the exact pareto set in multi-objective integer programming problems. Appl. Math. Comput. 219 (2013) 9652-9669.

[21] M. Mnif and S. Bouamama, Firework algorithm for multi-objective optimization of a multimodal transportation network problem. Proc. Comput. Sci. 112 (2017) 1670-1682.

[22] R.E. Moore, Methods and Applications of Interval Analysis. Vol 2 of Studies in Applied and Numerical Mathematics. SIAM, Philadelphia, PA (1979).

[23] H.G. Resat and M. Turkay, Design and operation of intermodal transportation network in the Marmara region of Turkey. Transp. Res. Part E: Logistics Transp. Rev. 83 (2015) 16-33. 
[24] J.R. Schott, Fault tolerant design using single and multi-criteria genetic algorithms. Masters Thesis, Massachusetts Institute of Technology (1995).

[25] Y. Sheng and Y. Gao, Shortest path problem of uncertain random network. Comput. Ind. Eng. 99 (2016) $97-105$.

[26] M. Steadieseifi, N.P. Dellaert, W. Nuijten, T. Van Woensel and R. Raoufi, Multimodal freight transportation planning: a literature review. Eur. J. Oper. Res. 233 (2014) 1-15.

[27] J.C. Thill and H. Lim, Intermodal containerized shipping in foreign trade and regional accessibility advantages. J. Transp. Geogr. 18 (2010) 530-547.

[28] X. Wang and Q. Meng, Discrete intermodal freight transportation network design with route choice behavior of intermodal operators. Transp. Res. Part B Methodol. 95 (2017) 76-104.

[29] L. Wang, L. Yang and Z.Y. Gao, The constrained shortest path problem with stochastic correlated link travel times. Eur. J. Oper. Res. 255 (2016) 43-57.

[30] R. Wang, K. Yang, L. Yang and Z.Y. Gao, Modeling and optimization of a road-rail intermodal transport system under uncertain information. Eng. App. Artif. Intell. 72 (2018) 423-436.

[31] H. Wei and M. Dong, Import-export freight organization and optimization in the dry-port-based cross-border logistics network under the Belt and Road Initiative. Comput. Ind. Eng. 130 (2019) 472-484.

[32] T. Yamada, B.F. Russ, J. Castro and E. Taniguchi, Designing multimodal freight transport networks: a heuristic approach and applications. Transp. Sci. 43 (2009) 129-143.

[33] S. Yan, L. Maoxiang and W. Danzhu, Bi-objective modelling for hazardous materials road-rail multimodal routing problem with railway schedule-based space-time constraints. Int. J. Environ. Res. Public Health 13 (2016) 1-31.

[34] X. Yang, J.M.W. Low and L.C. Tang, Analysis of intermodal freight from China to Indian Ocean: a goal programming approach. J. Transp. Geogr. 19 (2011) 515-527.

[35] K. Yang, L. Yang and Z. Gao, Planning and optimization of intermodal hub-and-spoke network under mixed uncertainty. Transp. Res. Part E: Logistics Transp. Rev. 95 (2016) 248-266.

[36] M.H.F. Zarandi, A. Hemmati, S. Davari and I.B. Turksen, Capacitated location-routing problem with time windows under uncertainty. Knowl.-Based Syst. 37 (2013) 480-489.

[37] R. Zhang, W.Y. Yun and I.K. Moon, Modeling and optimization of a container drayage problem with resource constraints. Int. J. Prod. Econ. 133 (2011) 351-359.

[38] J. Zhang, Q. Zhang and L. Zhang, A Study on the Paths Choice of Intermodal Transport Based on Reliability, in Z. Zhang, Z. Shen, J. Zhang, R. Zhang (eds) LISS 2014. Springer, Berlin-Heidelberg (2015) 305-315.

[39] Y. Zhang, P. Liu, L. Yang and Y. Gao, A bi-objective model for uncertain multi-modal shortest path problems. J. Uncertainty Anal. App. 3 (2015) 8.

[40] E. Zitzler and L. Thiele, Multiobjective evolutionary algorithms: a comparative case study and the strength Pareto approach. IEEE Trans. Evol. Comput. 3 (1999) 257-271. 